\title{
AAV-mediated overexpression of the CB1 receptor in the mPFC of adult rats alters cognitive flexibility, social behavior, and emotional reactivity
}

\author{
Matthias Klugmann ${ }^{1+}$, Anja Goepfrich ${ }^{2+}$, Chris M. Friemel ${ }^{2}$ and Miriam Schneider ${ }^{2 *}$ \\ Translational Neuroscience Facility, Department of Physiology, School of Medical Sciences, University of New South Wales, Sydney, NSW, Australia \\ 2 Research Group Developmental Neuropsychopharmacology, Institute of Psychopharmacology, Central Institute of Mental Health, University of Heidelberg, \\ Mannheim, Germany
}

Edited by:

Viviana Trezza, University "RomaTre," Italy

\section{Reviewed by:}

Maurice Elphick, Queen Mary

University of London, UK

Tiziana Rubino, University of Insubria, Italy

\section{${ }^{*}$ Correspondence:}

Miriam Schneider, Research Group

Developmental

Neuropsychopharmacology, Institute of Psychopharmacology, Central Institute of Mental Health, University of Heidelberg, J5, 68159 Mannheim, Germany.

e-mail:miriam.schneider@zi-

mannheim.de

${ }^{\dagger}$ Matthias Klugmann and Anja Goepfrich have contributed equally to this work.
The endocannabinoid (ECB) system is strongly involved in the regulation of cognitive processing and emotional behavior and evidence indicates that ECB signaling might affect these behavioral abilities by modulations of prefrontal cortical functions. The aim of the present study was to examine the role of the $\mathrm{CB} 1$ receptor in the medial prefrontal cortex (mPFC) on cognitive flexibility and emotional behavior. Therefore, the CB1 receptor was overexpressed by adeno-associated virus vector-mediated gene transfer specifically in the mPFC of adultWistar rats. Animals were then tested in different anxiety-related paradigms for emotional reactivity [e.g., elevated plus maze (EPM), light/dark emergence test (EMT), social interaction] and the attentional set shift task (ASST) - an adaptation of the human Wisconsin card sorting test - for cognitive abilities and behavioral flexibility. A subtle increase in exploratory behavior was found in CB1 receptor overexpressing animals (CB1-R) compared to Empty vector injected controls (Empty) in the EMT and EPM, although general locomotor activity did not differ between the groups. During social interaction testing, social contact behavior toward the unknown conspecific was found to be decreased, whereas social withdrawal was increased in CB1-R animals and they showed an inadequate increase in exploratory behavior compared to control animals. In the ASST, impaired reversal learning abilities were detected in CB1-R animals compared to controls, indicating reduced behavioral flexibility. In conclusion, upregulation of the CB1 receptor specifically in the rat $\mathrm{mPFC}$ induces alterations in emotional reactivity, leads to inadequate social behavior, and impairs cognitive flexibility. These findings might be relevant for neuropsychiatric disorders, since higher cortical CB1 receptor expression levels as well as similar behavioral impairments as observed in the present study have been described in schizophrenic patients.

Keywords: CB1 receptor, mPFC, cognitive flexibility, emotional behavior, social interaction, attentional set shift task

\section{INTRODUCTION}

The endocannabinoid (ECB) system has emerged in recent years as a key modulator of neuronal activity of various neurotransmitter systems and appears to be involved in synaptic plasticity in diverse brain structures. Accordingly, the ECB system and ECB signaling have been implicated in a variety of behavioral functions, including among others the regulation of emotional states, affective, and cognitive processes (for review see Viveros et al., 2005; Egerton et al., 2006; Pattij et al., 2008; Moreira et al., 2009).

One important brain region through which cannabinoids might exert their modulatory effects on cognition and emotional behavior is the prefrontal cortex (PFC). An abundant expression of CB1 receptors in this brain area (Herkenham et al., 1990; Mailleux and Vanderhaeghen, 1992; Marsicano and Lutz, 1999) indicates the significance of ECB signaling for the modulation of prefrontocortical neurotransmission (Egerton et al., 2006). It has been reported that systemic activation or blockade of cannabinoid CB1 receptors in the rat medial prefrontal cortex (mPFC) modulates emotional associative learning and memory formation, mainly through functional inputs from the basolateral amygdala (Laviolette and
Grace, 2006). Additionally, the importance of the ECB system for cognitive flexibility - a behavior that is highly dependent on prefrontocortical functions (Owen et al., 1991; Birrell and Brown, 2000; Egerton et al., 2005) - has been indicated in various studies in humans and rodents (for review see Egerton et al., 2006; Pattij et al., 2008). In humans, heavy marijuana use was shown to be associated with deficits in behavioral flexibility measured in a Wisconsin card sorting test (WCST; Bolla et al., 2002; Lane et al., 2007). Likewise, administration of cannabinoid agonists in laboratory rodents has also been found to impair cognitive flexibility in attentional set shifting paradigms - developed as an equivalent to the human WCST - (Egerton et al., 2005; Hill et al., 2006) and in an olfactory go/no-go discrimination task (Sokolic et al., 2011). It has been suggested that these cannabinoid effects might be related to the modulatory influence of ECB signaling on PFC neurotransmission (e.g., dopamine, GABA, and glutamate; Egerton et al., 2006; Pattij et al., 2008).

Beside this strong connection between prefrontocortical ECB signaling and cognitive functioning, the cortical ECB system appears to be also important for emotional reactivity (Valverde, 
2005; Viveros et al., 2005; Holmes and Wellman, 2009; Moreira et al., 2009). Stress- and anxiety-inducing stimuli consistently activate the PFC in rats (Singewald et al., 2003; Rubino et al., 2007), and in particular the mPFC appears to be an important region for anxiety-related behaviors (Holmes and Wellman, 2009). Lesions of the $\mathrm{mPFC}$ in rats have been found to induce anxiolytic-like effects in the elevated plus maze (EPM), social interaction and the shock-probe test (Shah and Treit, 2003). Additionally, an excitatory influence of corticotropin-releasing hormone in the mPFC has been suggested to modulate stress-induced HPA activity and anxiety-related behavior (Jaferi and Bhatnagar, 2007). With respect to ECB signaling in the PFC, experimental modulations of levels of the endocannabinoid anandamide (AEA), mainly by inhibition of the AEA degrading enzyme fatty acid amide hydrolase (FAAH) in the PFC, have been found to alter emotional behavior in rats (Rubino et al., 2008). While a strong decrease of AEA levels in the PFC by lentivirus-mediated overexpression of FAAH, was found to induce anxiogenic behavior, microinjections of the selective FAAH inhibitor, URB597, were found to induce anxiolytic responses at low doses and no effect or even an anxiogenic profile at higher doses (Rubino et al., 2008).

For the present study we were aiming to further examine the role of prefrontocortical ECB signaling on behavioral flexibility and emotional reactivity by region-specific overexpression of the $\mathrm{CB} 1$ receptor gene. Adeno-associated virus (AAV) gene transfer into a distinct brain region serves as an outstanding tool for studying gene function in complex behaviors of rodents (Klugmann and Szumlinski, 2008). We therefore employed the AAV-technology to overexpress the $\mathrm{CB} 1$ receptor gene in neurons of the mPFC of adult rats. The consequences of this manipulation on emotional behavior and cognition were investigated by a series of classical behavioral paradigms for emotional reactivity, including EPM, light/dark emergence test (EMT), and the social interaction test, and additionally, cognitive functions and behavioral flexibility were examined by the attentional set shift task (ASST).

\section{MATERIALS AND METHODS SUBJECTS}

Twenty-eight male Wistar ${ }^{\mathrm{TM}}$ Han rcc (Wistar) rats weighing 200$250 \mathrm{~g}$ were purchased from Harlan Laboratories (AN Venray, Netherlands). They were housed in groups of six in standard Makrolon $^{\mathrm{TM}}$ cages (Eurostandard type IV) under a 12/12-h lightdark cycle with the light phase starting at $8 \mathrm{am}$. During the light phase, a radio provided background noise. Animals had ad libitum access to tap water and standard lab chow if not indicated otherwise.

All experiments were conducted in accordance with the ethical guidelines for the care and use of laboratory animals, and were approved by local animal care committees (Sydney, Australia and Karlsruhe, Germany).

\section{AAV VECTOR PRODUCTION AND STEREOTAXIC DELIVERY}

The cDNA encoding the rat $\mathrm{CB} 1$ receptor was cloned into an AAV expression cassette containing the 1.1-kb CMV immediate early enhancer/chicken $\beta$-actin hybrid promoter (CBA), the woodchuck hepatitis virus post-transcriptional regulatory element (WPRE), and the bovine growth hormone polyadenylation sequence flanked by AAV2 inverted terminal repeats (pAAV-CB1). The same plasmid backbone with no cDNA was used as a control construct (pAAVEmpty). Of note, the CB1 cDNA (but not the AAV expression cassette) used in this study was identical to the one used in our previous work when we employed AAV-mediated overexpression of CB1 specifically in glutamatergic cells of the hippocampus and showed by GTPgammaS the biological functionality of the transgenic CB1 receptor (Guggenhuber et al., 2010). Packaging of AAV1/2 mosaic vectors with equal ratios of AAV1 and AAV2 capsid proteins was performed as described (Klugmann et al., 2005b). Briefly, using the standard $\mathrm{CaPO}_{4}$ precipitation method, HEK293 cells were transfected with the AAV plasmid, two helper plasmids encoding AAV1 and AAV2 rep and cap genes, and the adenoviral helper plasmid $\mathrm{pF} \Delta 6$. Cells were harvested $60 \mathrm{~h}$ after transfection, pellets lysed and vectors purified by heparin affinity chromatography. Genomic titers were determined by quantitative real-time PCR of vector genomes using primers against WPRE (During et al., 2003). For stereotaxic delivery of the AAV vector, adult rats were randomly assigned to treatment groups $(n=14)$ and control groups $(n=14)$. Animals were anesthetized with isoflurane ( $4 \%$ for induction and $1.5-2.5 \%$ for maintenance), administered via inhalation. The rats were then injected using $1.5 \mu \mathrm{l}$ of either AAV-Empty or AAV-CB1 $\left(6 \times 10^{11}\right.$ viral genomes $\left./ \mathrm{ml}\right)$ bilaterally into the $\mathrm{mPFC}(+2.7 \mathrm{~mm}$ $\mathrm{AP}, \pm 0.5 \mathrm{~mm} \mathrm{ML}, 4.5 \mathrm{~mm}$ DV from bregma), of adult rats based on established coordinates (Paxinos and Watson, 1998). Vector delivery was performed at a rate of $200 \mathrm{nl} / \mathrm{min}$ using a microprocessorcontrolled mini-pump (World Precision Instruments, Sarasota, FL, USA) with $33 \times \mathrm{G}$ beveled needles (World Precision Instruments) in a stereotaxic frame (Kopf Instruments, Tujunga, CA, USA). After the injection, the needle remained in place for two more minutes and was then carefully retracted in order to avoid vector backflow. Subsequently, the scalp was sutured and the rat was kept singly in a Makrolon ${ }^{\mathrm{TM}}$ cage (Eurostandard type III) until full recovery from anesthesia.

\section{IMMUNOHISTOCHEMISTRY}

The rostral-caudal extent of transgene expression of all animals used in behavioral experiments was assessed by $\mathrm{CB} 1$ immunohistochemistry. Animals not showing a robust pattern of transgene expression in the mPFC were excluded from the study. Rats were shortly anesthetized with a mixture of air and carbon dioxide $\left(\mathrm{CO}_{2}\right)$ and sacrificed by decapitation. Brains were quickly dissected, frozen on dry ice and stored at -80 until histological processing. Cryostatcut $14 \mu \mathrm{m}$ coronal sections were collected on Superfrost microscopic slides (Menzel GmbH \& Co KG, Braunschweig, Germany) before postfixing in 10\% buffered neutral formalin (SIGMA, Castle Hill, NSW, Australia). Then sections were rinsed with PBS containing $0.2 \%$ Triton X-100 (PBS-Triton), blocked in immunobuffer, (4\% horse serum in PBS, pH 7.4, with $0.4 \%$ Triton X-100) for $30 \mathrm{~min}$, and incubated overnight with a polyclonal anti-CB1 antiserum (1:2000; Cayman, Ann Arbor, USA). After washes, sections were incubated with anti-rabbit-Alexa488 antibody (1:1000, Molecular Probes, OR, USA). After two washes, the nuclear stain DAPI (Roche, Castle Hill, NSW, Australia) was administered for $5 \mathrm{~min}$, and sections coverslipped in Mowiol. Immunostaining was visualized using a BX51U epifluorescent microscope (Olympus, Tokyo, Japan). 


\section{BEHAVIORAL TESTING}

Behavioral testing began 3 weeks after vector infusion when AAV1/2mediated transgene protein expression had peaked to remain at stable levels (Klugmann et al., 2005a). Behavioral paradigms were conducted in the order listed below and animals were left undisturbed for at least 3 days between the different test sessions. The experimenter was blind to the treatment of the test subjects.

\section{Open field}

Locomotor activity was measured in an open field. The open field consisted of four equal arenas $(51 \mathrm{~cm} \times 51 \mathrm{~cm} \times 50 \mathrm{~cm})$ made of dark PVC. Distance traveled $(\mathrm{cm})$ was recorded for $30 \mathrm{~min}$ at a light intensity of $50 \mathrm{~lx}$. For the analysis of locomotor activity the observation program Viewer ${ }^{2}$ (Biobserve GmbH, Bonn, Germany) was used. Animals were habituated 1 day before testing to the new environment for $10 \mathrm{~min}$.

\section{Light/dark emergence test}

The EMT took place in a light/dark box which consisted of two different compartments, separated by a dividing wall with a $10-\mathrm{cm} \times 15-\mathrm{cm}$ wide opening which enabled the test subjects to move freely between the compartments. The first compartment, with black walls $(25 \mathrm{~cm} \times 25 \mathrm{~cm} \times 40 \mathrm{~cm})$ could be closed by a lid and was used as start box. The second compartment had gray walls $(25 \mathrm{~cm} \times 50 \mathrm{~cm} \times 40 \mathrm{~cm})$ and was brightly illuminated $(90 \mathrm{~lx})$. Rats were initially placed for $1 \mathrm{~min}$ in the dark, closed compartment and their behavior was recorded for $5 \mathrm{~min}$ after the start box was opened. Subsequent video analysis by a trained experimenter scored the latency of the animals to emerge from the dark compartment into the light compartment (s) (an entry was defined when the animal entered the compartment with all four limbs), the emergence frequency, the duration of time spent in the light compartment (s), the amount of rearings and risk assessment behavior (only head or forepaws are placed in the open compartment without concomitant movement of the hindlimbs, even if the rat subsequently entered the area). The apparatus was thoroughly cleaned with 70\% ethanol between the sessions.

\section{Elevated plus maze}

The EPM consisted of a plus-shaped apparatus made of dark gray PVC elevated $50 \mathrm{~cm}$ above the floor with two open arms $(12 \mathrm{~cm} \times 50 \mathrm{~cm} \times 50 \mathrm{~cm})$ which were illuminated by $80 \mathrm{~lx}$ and two enclosed arms $(12 \mathrm{~cm} \times 50 \mathrm{~cm} \times 50 \mathrm{~cm})$. All arms extended from a central square $(10 \mathrm{~cm} \times 10 \mathrm{~cm})$. At the beginning of each trial, rats were placed in a closed arm of the EPM. Each rat was videotaped for $5 \mathrm{~min}$ and the following behaviors were analyzed: number of entries into open or closed arms (an entry was defined if all four paws were placed on that arm), time spent in open and closed arms (s), head dips (the whole head is lowered beneath the edge of an open arm), risk assessment (only head or forepaws are placed in an open arm without concomitant movement of the hindlimbs, even if the rat subsequently entered the arm), self grooming and self-grooming time (s). Percentage of open arm entries [open arm entries/(open + closed arm entries $) \times 100]$ and percentage of time spent in open arms [open arm time/(open + closed arm time $\times 100$ ] were calculated as well. The apparatus was thoroughly cleaned with $70 \%$ ethanol between the sessions.

\section{Social interaction test}

Social interaction with an unfamiliar social partner (7- to 8-weekold male Wistar rat) was assessed in an open field for $5 \mathrm{~min}$ as described before (Schneider et al., 2008; Waltereit et al., 2011). The following behavioral elements were quantified only for the experimental rat: (1) Social behavior, including contact behavior (grooming and crawling over), social exploration (anogenital and non-anogenital investigation), and approach/following; (2) social evade was scored as an active withdrawal from social contact; and (3) self-grooming behavior (for detailed description see Schneider et al., 2008).

\section{Attentional set shift test}

Apparatus. The test apparatus was made of dark gray PVC consisting of a small start compartment $(20 \mathrm{~cm} \times 20 \mathrm{~cm} \times 40 \mathrm{~cm})$ adjacent to the test compartment $(40 \mathrm{~cm} \times 50 \mathrm{~cm} \times 40 \mathrm{~cm})$. The two compartments were separated by a sliding door of $20 \mathrm{~cm}$ width. Two small ceramic pots (diameter $7 \mathrm{~cm}$, depth $4 \mathrm{~cm}$ ) were positioned into the test compartment $16 \mathrm{~cm}$ apart from each other and separated by a solid divider ( $20 \mathrm{~cm}$ length). One of the cups was baited with a casein pellet (Bio Serve Dustless Precision Pellets ${ }^{\varpi}$, Bilaney, Kent, UK). The bowls were filled with different digging materials that were scented and the food reward was deeply buried into one of the pots. Rats were trained to dig in the bowls to retrieve the rewards. The presence or absence of the reward pellet in the digging bowl was targeted by either an olfactory (odor of digging medium) or a visual-tactile cue (shape and tactile quality of digging medium).

Habituation. Animals were familiarized with the food reward, the ceramic pots, and different digging materials prior to testing. During 1-2 nights, the pots were filled with homecage bedding and casein pellets were placed on top and buried in the bowls. The pots were rebaited several times and left in the homecage overnight (not more than three pots per cage). The following nights, some of the digging materials were introduced in the same manner. On the second day of habituation, two familiar animals were placed into the test apparatus and allowed to freely explore the entire test box for $15 \mathrm{~min}$. Afterward, the rats were returned to the homecage. At the next day, each rat was placed into the apparatus individually for a 15 -min habituation period. Figure 1 illustrates the timeline of habituation and testing procedure. During the complete period of ASST testing (including the habituation period) all animals were maintained on approximately $90 \%$ of their free-feeding bodyweight by applying a mild food restriction schedule (12 g chow/rat/day).

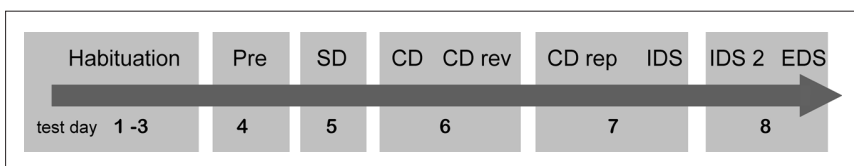

FIGURE 1 |Timeline of the ASST paradigm. Pre: pretraining; SD: simple discrimination; CD: compound discrimination; CDrev: compound discrimination reversal; CDrep: compound discrimination repetition; IDS; intradimensional shift; EDS: extradimensional shift. 
Testing procedure. The testing procedure was adapted from Birrell and Brown (2000; see also Colacicco et al., 2002; Egerton et al., 2005; Figure 1). After habituation, rats were subjected to a pretraining schedule. Therefore, animals had to retrieve the reward from Empty pots in the apparatus and subsequently from pots filled with digging medium. First, the reward was placed on the top of the digging medium and in following trials the pellet was gradually buried deeper in the digging material. The rats had to retrieve the reward five times within 2 min trials and then four times within $1 \mathrm{~min}$ trials. As soon as the rat retrieved the pellet or the trial time expired, the animal was gently pushed by the experimenter into the waiting compartment. The pots were rebaited during an intertrial interval of $30 \mathrm{~s}$ during which the rat waited in the start compartment until the sliding door was lifted again for the next trial. After the test session the rat was returned to its homecage. Material from the pretraining was not used again in later testing stages.

For the training sessions, eight common spices: capsicum, cumin, basil, thyme, rosemary, nutmeg, dill, and cardamom were used as odor stimuli. The digging media were colored and black silica sand, beech chipping, pine bark, cork granules, hamster bedding, straw pellets, and rough stones (see Table 1). The digging media were mixed with the spices and additional casein pellet powder was intermixed in order to exclude the possibility of olfactory reward detection.

In all training sessions, a criterion of six consecutive correct trials was used for successful learning (trials to criterion). This method was applied for all subsequent training trials. For the simple discrimination (SD) task, each rat was presented two bowls containing scented digging medium with the same odor but different media. The visual/tactile stimulus dimension indicated the position of the reward during SD testing and therefore, rats had to learn that only the bowl with a certain medium contained the food pellet. For the compound discrimination $(\mathrm{CD})$, which was tested 1 day later, an additional odor was introduced and used together with the two familiar digging media and the previous odor. In this training stage, the digging media could be paired with one of the two odors respectively. However, still the previously baited digging medium used during $\mathrm{SD}$ - indicated the location of the reward during this stage, independent from the two odors. In the next session (on the same day), the previously learned rule was reversed (CD reversal, CDrev). The medium that had previously been incorrect was now associated with the food reward and accordingly, the unrewarded sets became baited. On the following day, a repetition test (CD repetition, CDrep) ensured that the animals had not forgotten the rules of the CDrev. In the following test session, the intradimensional shift (IDS), a set of new complex stimuli was introduced,

Table 1 | Examples of odor-medium pairs employed in the ASST.

\begin{tabular}{llll}
\hline Digging medium & Digging medium & Odor & Odor \\
\hline $\begin{array}{l}\text { Seramis } \\
\text { Colored silica }\end{array}$ & Hamster bedding & Cumin & Capsicum \\
sand $(3-4 \mathrm{~mm})$ & & & \\
Beech chipping & Rough stones & Nutmeg & Basil \\
Straw pellets & Pine bark & Thyme & Dill \\
Cork granules & Black silica sand & Rosemary & Cardamom \\
& $(1-2 \mathrm{~mm})$ & &
\end{tabular}

and the rat had to discriminate the baited from the unbaited cup by attending at the same perceptual dimension (digging material) as in the previous training. The subsequent day, a new set of complex stimuli was introduced and the rat had to apply the same rule (IDS2). In the last test session, again a new set of complex stimuli was presented but this time a cue of the previously irrelevant perceptual dimension predicted the reward (extradimensional shift, EDS). Therefore, not the type of digging material predicted the reward any longer, but the odor was relevant to obtain the reward.

If an animal stopped responding for several trials during a test session it was returned to the homecage for up to $1 \mathrm{~h}$ before resuming the test again. In this case, the sum of the number of trials was taken.

\section{STATISTICAL ANALYSIS}

Differences between CB1-R and Empty vector expressing animals for locomotor activity, EPM and EMT performance as well as social interaction testing were analyzed by Student's $t$-tests. Performance in the ASST was analyzed by MANOVA. The overall performance in the ASST between the groups was compared and specified by Wilk's $\lambda$, whereas learning differences at each ASST stage were calculated with multiple ANOVAs.

All data are expressed as mean \pm SEM. The overall level of statistical significance was defined as $p<0.05$.

\section{RESULTS}

\section{HISTOLOGICAL ANALYSIS}

After completion of behavioral testing (4 month after infusion), the gene transfer efficacy was determined by immunohistochemical analysis using an antibody against $\mathrm{CB} 1$ receptors. Abundant ectopic $\mathrm{CB} 1$ receptor immunoreactivity could be detected specifically in the mPFC of AAV-CB1-injected animals including prelimbic, infralimbic, and cingulate cortical areas (Figures $2 \mathrm{~A}-\mathrm{C}$ ). Consistent with our previous studies using the same AAV serotype but different transgenes (Lominac et al., 2005), the rostro-caudal extension of the vector spread was observed $1 \mathrm{~mm}$ around the injection site. In comparison, immunoreactivity of endogenous $\mathrm{CB} 1$ in AAVEmpty treated brains revealed by increased exposure time was more homogenous and less abundant (Figures 2D-F) indicating anatomically correct and efficient gene delivery. At higher magnification, ectopic $\mathrm{CB} 1$ receptor expression was visualized in neuronal soma and the neuropil (Figures $2 \mathrm{~A}^{\prime}-\mathrm{C}^{\prime}$ ). Inconsistent or low CB1 receptor expression was detected in the mPFC of two animals and these animals were therefore excluded from further analysis.

\section{LOCOMOTOR ACTIVITY}

No significant differences were detected between CB1-R rats and Empty animals for locomotor activity in an open field (Figure 3). Both groups did not differ in distance traveled $(\mathrm{cm})$ during the 30 - $\mathrm{min}$ test period (Student's $t$-test, $p>0.05$; CB1-R: $n=12$; Empty: $n=14$ ).

\section{LIGHT/DARK EMT}

No significant differences were detected in the light/dark EMT between CB1-R and Empty animals for emergence latency, risk assessment, time spent in the lit compartment and rearing (Student's $t$-test, $p>0.05)$. However, statistical analysis revealed a strong trend $(p=0.056)$ for a higher emergence frequency of CB1-R rats compared to controls (Table 2; CB1-R: $n=12$; Empty: $n=14$ ). 

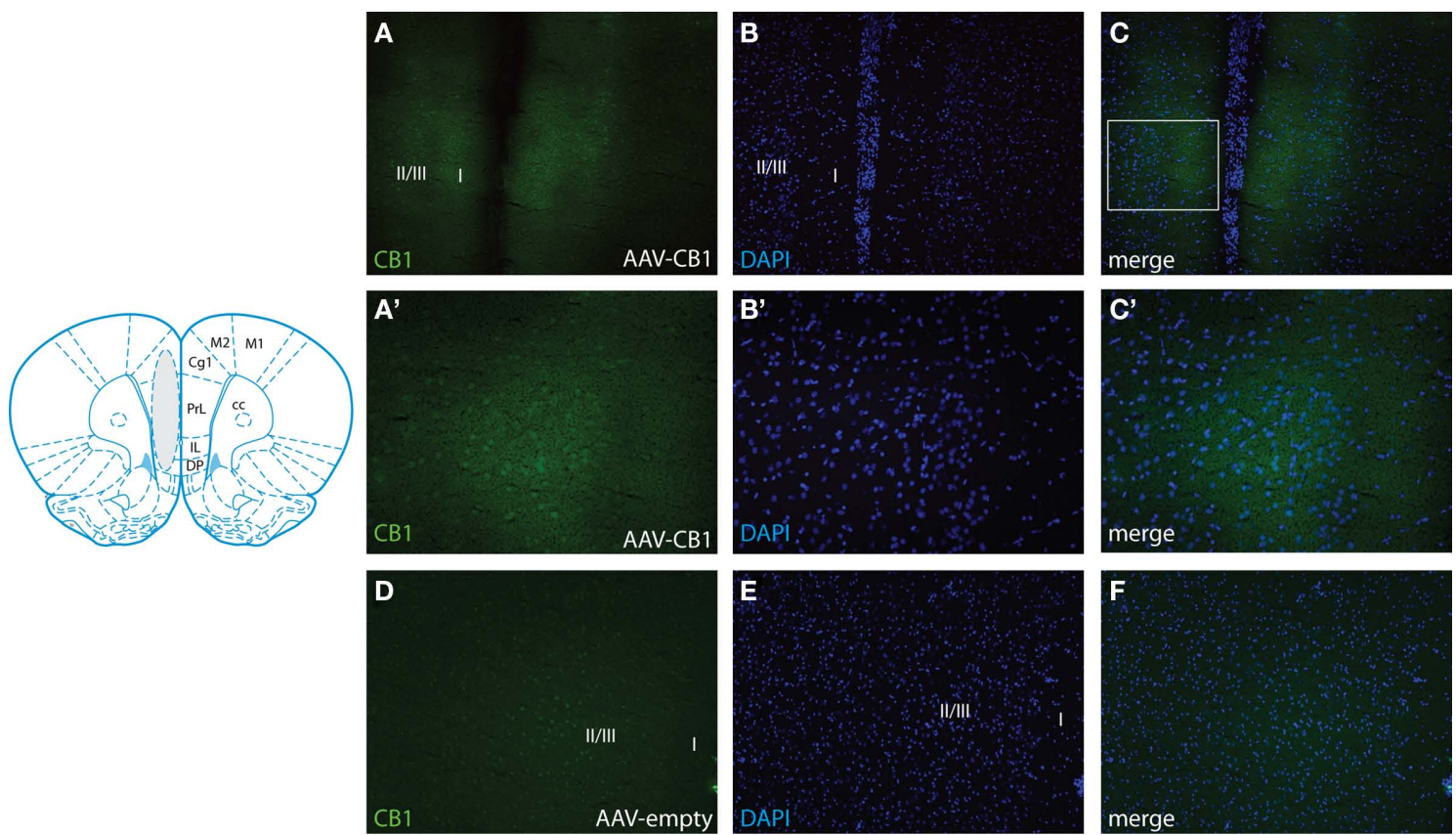

FIGURE 2 |Adeno-associated virus-mediated CB1 expression in the MPFC. The cartoon (adapted from Paxinos and Watson, 1998) shows the representative transduced area (gray) in animals injected with AAV-CB1. Representative immunostaining for $\mathrm{CB} 1$ in the mPFC of AAV-CB1-injected rats at low (10 $\times$ for A-C) and high (20x for $\left.\mathbf{A}^{\prime}-\mathbf{C}^{\prime}\right)$ magnification. (A) Extent of CB1 immunoreactivity showing robust transduction in the target area. (B) Same section stained for the nuclear stain DAPI. (C) Overlay of (A) and (B). The box in (C) indicates the area magnified in $\left(\mathbf{A}^{\prime} \mathbf{-} \mathbf{C}^{\prime}\right)$. Note that adjustment of the exposure time for visualization of ectopic CB1 precludes visualization of endogenous CB1. The counterstaining

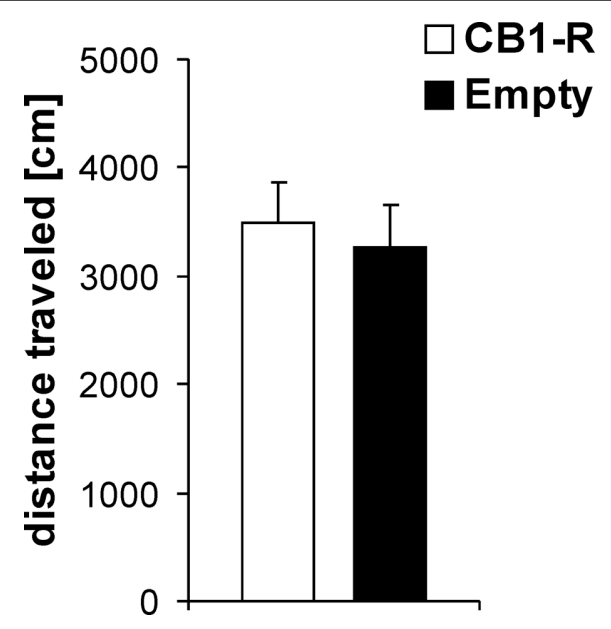

FIGURE 3 | Locomotor activity in an open field. No significant differences were found between CB1 receptor overexpressing animals (CB1-R) and Empty vector injected controls (Empty). Values are expressed as mean \pm SEM.

\section{ELEVATED PLUS MAZE}

Similar as for the EMT, only subtle differences were detected between CB1-R rats and Empty controls in the EPM (Table 3). The two groups did not differ for time in open and closed arms, with DAPI delineates the tissue and shows correct targeting of ectopic CB1 expression (C). (C') High power micrograph showing CB1 immunoreactivity in the neuronal soma and neuropil of the mPFC. (D) Representative brain section (left hemisphere) of an AAV-Empty-injected animal showing endogenous CB1 immunoreactivity at low power (10x). Note that the exposure time was $2.5 x$ more than for the visualization of transgenic CB1 shown in (A). (E) DAPI stain. (F) Merger of (D) and (E). Prl, prelimbic cortex; $\mathrm{Cg} 1$, area 1 of the cingulate cortex; IL, infralimbic cortex; DP, dorsal peduncular cortex; M1/2, motor cortex; cc, corpus callosum; I, II, III, cortical layers.

Table 2 | Light/dark EMT performance in CB1-R rats and Empty control animals.

\begin{tabular}{lcc}
\hline EMT & Empty & CB1-R \\
\hline Emergence frequency & $3.0( \pm 0.5)$ & $4.83( \pm 0.7)^{\#}$ \\
Emergence latency $(\mathrm{s})$ & $126.6( \pm 22.4)$ & $81.3( \pm 16.3)$ \\
Rearing & $5.8( \pm 1.7)$ & $8.9( \pm 1.2)$ \\
Risk assessment & $9.0( \pm 1.2)$ & $10.4( \pm 0.8)$ \\
Time in lit compartment $(\mathrm{s})$ & $47.8( \pm 13.1)$ & $70.1( \pm 9.2)$ \\
\hline
\end{tabular}

Values are expressed as mean \pm SEM $(p<0.1$ is indicated by \#).

percentage of time spent in open arms, open arm entries, percentage of open arm entries, head dips, risk assessment, and self grooming (Student's $t$-test, $p>0.05$ ). However, a strong increase in closed arm entries was detected in rats overexpressing the CB1 receptor $(p=0.009$; CB1-R: $n=12$; Empty: $n=14)$.

\section{SOCIAL INTERACTION TEST}

Several behavioral differences between CB1-R rats and controls were observed during social interaction testing (Figure 4). CB1-R animals engaged significantly more in anogenital exploration (Student's $t$-test, $p=0.009)$ and approach/following $(p=0.032)$ during interaction with the unfamiliar social partner compared to Empty animals. Additionally, CB1-R rats showed significant lower 
social contact behavior compared with the Empty group $(p=0.013)$. Finally, CB1-R animals were found to withdraw significantly more often from social contact if initiated by the social partner (social evade; $p=0.024$ ). No significant differences between the groups were detected for non-anogenital exploration and self-grooming behavior (Student's $t$-test, $p>0.05$; CB1-R: $n=12$; Empty: $n=14$ ).

\section{ATTENTIONAL SET SHIFT TASK}

All animals learned to perform a series of six consecutive correct trials at each stage of the set shifting paradigm. No differences in initial reward consumption were detected during habituation between CB1-R and Empty animals (data not shown). Over the

Table 3 | Elevated plus maze performance in CB1-R rats and Empty control animals.

\begin{tabular}{lcc}
\hline EPM & Empty & CB1-R \\
\hline Open arm time $(\mathrm{s})$ & $25.6( \pm 8.8)$ & $35.2( \pm 8.9)$ \\
Closed arm time $(\mathrm{s})$ & $250.6( \pm 15.4)$ & $231.4( \pm 16.7)$ \\
Open arm time $(\%)$ & $10.3( \pm 3.7)$ & $14.1( \pm 3.7)$ \\
Open arm entries & $2.1( \pm 0.7)$ & $2.3( \pm 0.5)$ \\
Closed arm entries & $6.4( \pm 0.8)$ & $9.5( \pm 0.7)^{*}$ \\
Open arm entries $(\%)$ & $18.4( \pm 5.7)$ & $16.8( \pm 3.1)$ \\
Head dips & $7.4( \pm 1.7)$ & $6.6( \pm 1.0)$ \\
Risk assessment & $7.7( \pm 1.0)$ & $9.4( \pm 0.7)$ \\
Self grooming & $0.6( \pm 0.2)$ & $0.4( \pm 0.1)$
\end{tabular}

Values are expressed as mean \pm SEM ( $p<0.05$ is indicated by asterisks).

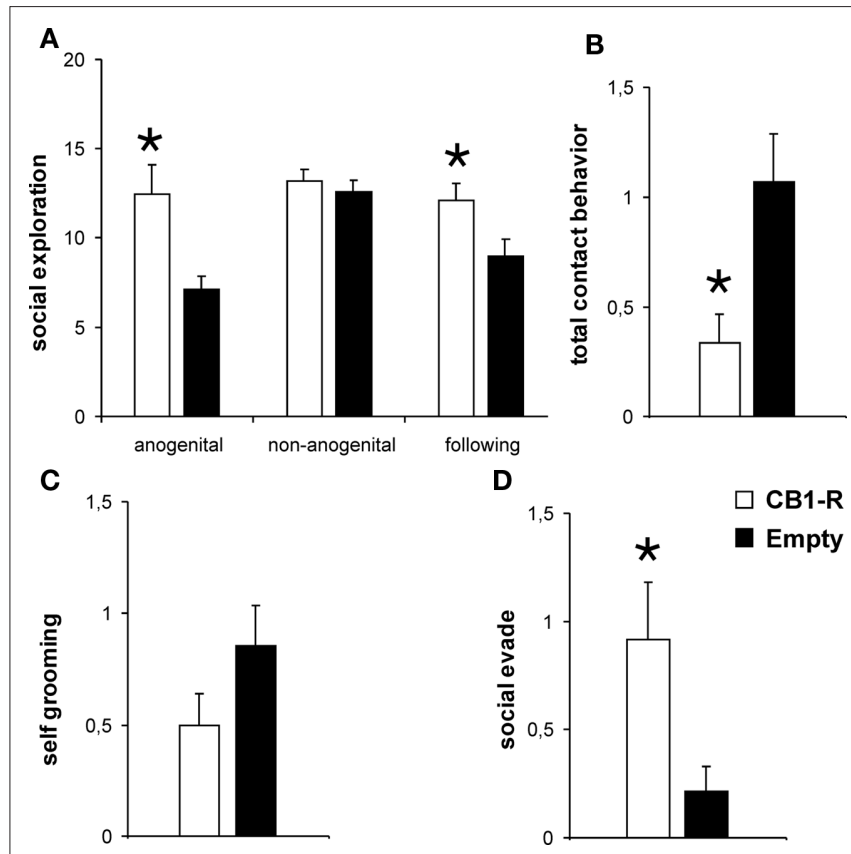

FIGURE 4 | Behavioral performance during social interaction with an unknown social partner. Significant differences between CB1-R and Empty animals were detected for anogenital exploration, approach/following (A), social contact behavior (B) and evade upon social contact (D). No differences were observed for non-anogenital exploration (A) and self-grooming behavior (C). Values are expressed as mean \pm SEM ( $p<0.05$ is indicated by asterisks). course of the whole experiment animals needed gradually less trials to reach the learning criterion for pretraining, SD and CD as well as for IDS and IDS2. All rats needed more trials to criterion to successfully complete the CD reversal and the EDS. The higher number of trials to criterion indicates that (a) CDrev and EDS were more challenging than the others stages (see also Barense et al., 2002; Colacicco et al., 2002) since subjects had formed attentional sets, and (b) that the set shift test worked properly and rats learned the cognitive task (Figure 5). MANOVA analysis revealed an overall significant difference between CB1-R and Empty control animals for the ASST (Wilk's $\lambda=0.14 F_{8,16}=2.9 p=0.035$ ). Further analysis by multiple ANOVAs indicated a significant difference between CB1-R and Empty control animals only for the CDrev stage $\left(F_{1,23}=7.4\right.$ $p=0.012)$, where CB1-R animals required more trials to criterion than Empty animals. No statistical differences between CB1-R and Empty control rats were found in the performance at all other stages ( $p>0.05$; CB1-R: $n=12$; Empty: $n=14$ ).

\section{DISCUSSION}

Local overexpression of the $\mathrm{CB} 1$ receptor by AAV-mediated gene transfer in the $\mathrm{MPFC}$ (including prelimbic, infralimbic, and anterior cingulate regions) of adult rats was found to alter emotional reactivity and social behavior and induce a deficit in cognitive flexibility. We detected subtle differences during testing of classical anxiety-related behavioral paradigms - EPM and light/dark EMT - between CB-R rats and Empty controls, which were mainly related to activity levels in these anxiety-inducing environments. Additionally, inadequate social behavior and social withdrawal were observed after cortical CB1 receptor overexpression during interaction with an unfamiliar conspecific. Finally, CB1-R rats showed a deficit specifically in reversal learning during the ASST.

The PFC is an essential brain region for higher-order cognitive functions and emotional processing, mainly due to its important integral role for the selection and processing of information necessary to plan, control and direct behavior according to changing environmental demands (Holmes and Wellman, 2009). CB1 receptors are abundantly expressed in the PFC (Herkenham et al., 1990; Mailleux and Vanderhaeghen, 1992; Marsicano and Lutz, 1999), strongly indicating an involvement of ECB signaling for

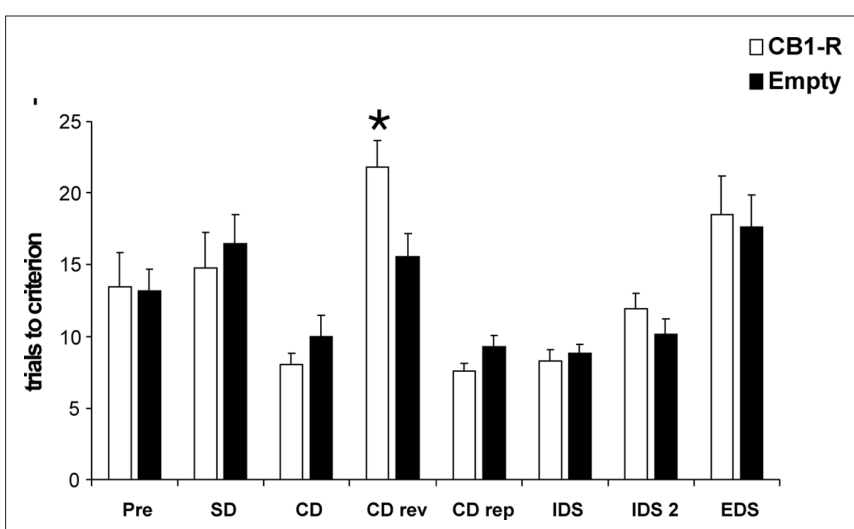

FIGURE 5 | Behavioral performance in the ASST. CB1-R rats differed significantly from Empty animals in the CDrev stage. Values are expressed as mean \pm SEM ( $p<0.05$ is indicated by asterisks). 
the modulation of prefrontocortical-mediated behaviors (Egerton et al., 2006). CB1 receptors are expressed by GABAergic interneurons and by pyramidal neurons in the PFC (Marsicano and Lutz, 1999) and ECBs appear to play a key role in the induction of LTD at PFC synapses of evoked and spontaneous excitatory postsynaptic currents recorded in layer V/VI pyramidal neurons (Lafourcade et al., 2007). A plethora of data indicates differences in the function of the CB1 receptor in glutamatergic versus GABAergic cells with respect to the brain region and experimental paradigms. These data are obtained mainly from pharmacological studies or genetic studies using conditional mouse mutants with cell-type-specific CB1 receptor ablation (e.g., Azad et al., 2008; Steiner et al., 2008; Massa et al., 2010). Only recently, we could achieve AAV-mediated conditional overexpression of $\mathrm{CB} 1$ receptors in glutamatergic hippocampal neurons (Guggenhuber et al., 2010). However, this approach depends on the availability of transgenic animals expressing cre-recombinase under tissue specific promoters and is therefore limited so far to mice. For the present study we have employed a neurotropic AAV vector system known to transduce all types of neurons at similar efficiency (Guggenhuber et al., 2010). The major difference to the genetic studies using germ line transgenics is that animals subjected to AAV-mediated CB1 receptor delivery in this study had a normal development into adulthood and the somatic gene transfer was highly specific to a confined brain area (mPFC). A single administration of AAV-CB1 results in long-term and stable transgene expression (Klugmann et al., 2005a) and to our knowledge this is the first study to demonstrate behavioral effects after persistent overexpression of the $\mathrm{CB} 1$ receptor in the adult rat PFC. However, despite the extraordinary spatio-temporal control that is achieved by employing the AAV-technology, one must be cautious to what extent conclusions can made about the role of endogenous CB1 receptors in physiological/behavioral processes based on an approach where protein levels are artificially elevated. In fact, since the tropism of the AAV $1 / 2$ serotype used in this study is pan-neuronal (Richichi et al., 2004; Guggenhuber et al., 2010), it is likely that GABAergic and glutamatergic neurons in the targeted PFC were transduced with equal efficacy. By elevating CB1 expression in the $\mathrm{mPFC}$, neural activity in this brain region is likely to be affected in various ways, perhaps by reducing glutamatergic transmission or GABAergic transmission, most likely both. Importantly, the protein levels of endogenous CB1 are 20-30-fold enriched in GABAergic over glutamatergic neurons, so it is conceivable that the introduction of comparable absolute amounts of ectopic CB1 will yield very different levels of relative overexpression in these different types of neurons. This consideration suggests that overexpression may be supraphysiological in glutamatergic but not in GABAergic cells.

\section{EMOTIONAL BEHAVIOR}

Although, the ECB system has been shown to act as an important modulator of emotional behavior and emotional reactivity (Valverde, 2005; Viveros et al., 2005; Moreira et al., 2009) and especially ECB signaling in the PFC has been implicated in these behaviors (Rubino et al., 2007, 2008), we did not observe profound alterations in anxiety-related behavior after overexpression of the $\mathrm{CB} 1$ receptor in the $\mathrm{mPFC}$ in the light/dark EMT and the EPM. Statistical differences compared to Empty controls were only detected in emergence frequency into the lit compartment (EMT) and the number of closed arm entries (EPM), which were both increased in CB1-R animals. Therefore, overexpression of the CB1 receptor in the $\mathrm{MPFC}$ appears to stimulate locomotor activity in anxiogenic environments. Since we did not observe any differences between the testing groups for normal activity in an open field under non-anxiogenic conditions (low lux and familiar environment), this hyperlocomotion and increased exploratory behavior seems to be linked exclusively to emotionally challenging environments, without affecting anxiety-related behaviors per se.

The involvement of ECB signaling in the mediation of anxietyrelated behaviors is very complex and only partially understood. In animal studies cannabinoids have been shown to induce anxiogenic as well as anxiolytic-like responses, depending upon dosage, behavioral tests used, the context, species, or genetic strain (Valverde, 2005; Viveros et al., 2005). CB1 receptor deficient mice have been shown to display anxiogenic-like responses in different behavioral paradigms, such as EPM, light/dark box, and open field (Haller et al., 2002; Viveros et al., 2005). Accordingly, in FAAH knockout mice, where AEA levels are increased, reduced anxiety-related behaviors have been reported both in the EMP and in the light/ dark EMT test. These genotype-related differences were prevented by the CB1 receptor antagonist SR141716 (Moreira et al., 2008).

With respect to prefrontocortical effects of cannabinoids on emotional behavior, it has been demonstrated recently that the endocannabinoid AEA in the PFC appears to be an important modulator of anxiety-related behaviors (Rubino et al., 2008). Administration of methanandamide (a metabolically stable analog of AEA) directly into the PFC revealed anxiolytic-like responses in rats in the EPM test for low doses, whereas high doses induced anxiogenic effects. In line with this, a marked decrease of AEA levels in the PFC, achieved by lentivirus-mediated local overexpression of FAAH, produced an anxiogenic response, supporting an anxiolytic role for a physiological increases in AEA in the PFC (Rubino et al., 2008).

In the present study we did not observe a clear anxiolytic response. Our data indicate that increased expression of the CB1 receptor in the $\mathrm{mPFC}$ does not affect overall anxiety-related behaviors, but increases arousal and locomotor response in anxiogenic environments, probably through interaction with the amygdala. An important role for CB1 receptors within the amygdala-prefrontal cortical circuit has been suggested for heightened emotional processing since $\mathrm{CB} 1$ receptor activation was found to potentiate the encoding of emotional associative learning at the level of single mPFC neurons (Laviolette and Grace, 2006). Further studies will have to examine how $\mathrm{AAV}$-mediated $\mathrm{CB} 1$ receptor overexpression in the $\mathrm{mPFC}$ affects AEA levels or FAAH activity within the mPFC and other prefrontal or subcortical regions.

\section{SOCIAL INTERACTION}

The social interaction test is an ethologically based test that measures explorative and social behavior between two rodents meeting for the first time in an open field and has been suggested as a measure for anxiety-related behaviors (File and Hyde, 1978). For the present study the test was used to assess social behaviors in CB1 receptor overexpressing animals and controls in an emotionally arousing context. Various differences between CB1-R rats and 
Empty controls were detected during social interaction testing. A higher number of anogenital exploration and approach/following behavior was observed in CB1-R animals. Additionally, prefrontocortical overexpression of the CB1 receptor increased the number of active social evade upon contact of the social partner and decreased social contact behaviors (grooming and crawling over/ under). Similar, as observed during the EPM and EMT, CB1-R animals appeared to be more active and showed increased exploratory behavior toward the unfamiliar conspecific during social interaction testing, compared to control animals. Despite this high and inadequate increase in social exploration, CB1-R animals were found to avoid normal social contact, especially when the contact was initiated by the social partner.

It is well known that the PFC is involved in the modulation of social behaviors and social skills (De Bruin, 1990; Wood, 2003) and we could show in a previous study that neonatal mPFC lesions in rats decreased social contact behavior persistently in adulthood (Schneider and Koch, 2005). A clear involvement of the ECB system and cannabinoids in social behaviors during development and adulthood has been demonstrated before for social interaction, social recognition, homecage social behavior, and social play behavior (e.g., Sieber, 1982; Schneider and Koch, 2002, 2005; Schneider et al., 2008; Trezza and Vanderschuren, 2008). With respect to social interaction testing it has been shown that administration of $\Delta$ 9-tetrahydrocannabinol (THC; Sieber et al., 1980; Van Ree et al., 1984), as well as adolescent cannabinoid exposure (O'Shea et al., 2004) reduces social interaction in rodents. Additionally, reduced social interaction has also been described in CB1 receptor knockout mice (Haller et al., 2004). However, in most of these studies all social behaviors during social interaction testing were summed up to a single social interaction score and therefore no information is given on possible changes in particular behaviors (e.g., social exploration versus social contact behavior). We have found recently that acute treatment with the cannabinoid agonist WIN 55,212-2 (WIN) attenuates social exploratory behavior in adult rats. However, chronic pubertal WIN treatment was found to persistently decrease social contact behavior and to increase anogenital exploration and social withdrawal during social interaction testing and homecage recording (Schneider et al., 2008). Cannabinoid effects on active evade from social contact were also described in baboons, where THC was found to induce social withdrawal and isolation (Sieber, 1982). This is in line with our present observation on increased social evade upon social contact in CB1-R animals, although behavioral effects of cannabinoid pharmacology (in particular if administered systemically) can not be considered equivalent with the persistent and region-specific overexpression of the $\mathrm{CB} 1$ receptor in the $\mathrm{mPFC}$.

\section{BEHAVIORAL FLEXIBILITY IN THE ASST}

Behavioral flexibility is an important cognitive skill for survival of an individual, since it enables an organism to successfully adapt to changing environments and circumstances, and requires the capacity to adjust behavioral strategies and to suppress "previous" whilst initiating "new" response patterns (Pattij et al., 2008). For the present study behavioral flexibility was assessed with the ASST - developed as an equivalent to the human WCST (Birrell and Brown, 2000). The ASST involves a series of compound perceptual discriminations that require subjects either to maintain attention and discriminate between two stimuli within one modality or dimension (IDS), or shift the attention between two stimuli from two different modalities or dimensions (EDS). CB1-R animals were found to show impaired learning in the CD reversal stage compared to Empty controls, whereas no significant differences were observed for other training stages. Our data indicate that overexpression of $\mathrm{CB} 1$ receptors in the $\mathrm{mPFC}$ does neither affect intradimensional or extradimensional set shifting abilities but impairs reversal learning of a previous rule. While extradimensional (attentional) set shifting ability serves as a measure of the capacity to shift attentional bias between different perceptual features of complex stimuli, reversal learning requires the capacity to update associations (to form new associations and at the same time inhibit those previously learned) between exteroceptive stimuli and reinforcement presentation when the contingencies between stimuli and reward presentation are reversed (Egerton et al., 2005).

An important role of the ECB system in these attentional and adaptational cognitive functions has been suggested by various studies in humans and rodents (for review see Egerton et al., 2006; Pattij et al., 2008). In humans, heavy marijuana use was shown to be associated with deficits in behavioral flexibility measured with the WCST (Bolla et al., 2002; Lane et al., 2007). Likewise, administration of cannabinoid agonists in laboratory rodents has also been found to impair cognitive flexibility in the ASST paradigm (Egerton et al., 2005), a cross maze task (Hill et al., 2006) and in an olfactory go/no-go discrimination task (Sokolic et al., 2011). Acute administration of THC impaired performance on the ASST when rats were required to reverse stimulus reward associations or shift cognitive set between stimuli belonging to the same perceptual dimension (IDS). In contrast, the ability to shift attentional set between perceptual dimensions (EDS) was unaffected by THC administration (Egerton et al., 2005). These results are partially in line with our present findings where persistent CB1 receptor overexpression impaired reversal learning without affecting EDS, although we did not detect additional effects on IDS in our CB1-R animals. Egerton et al. (2005) concluded from their findings that acute THC administration might selectively increase rigidity in the processes required to update responses based on affective associations between stimuli and reward presentation, but does not affect higher-order attentional flexibility. By testing attentional set shift abilities in a cross maze task, Hill et al. (2006) detected that administration of a high dose of the $\mathrm{CB} 1$ receptor agonist $\mathrm{HU}-210$ consistently increased the tendency for rats to perseverate when shifting from a response to a visual-cue-based discrimination and vice versa, whereas a low dose of HU-210 elicited an opposite behavioral profile, with reliable reductions in perseverative errors. Additionally, systemic administration of a low dose of the CB1 receptor antagonist AM251 facilitated set shifting by reducing the number of perseverative errors. The cross maze task differs in many aspects from the ASST paradigm applied by Egerton et al. (2005) and therefore the outcome of both studies is difficult to compare. While deficits observed after CB1 receptor activation in the Hill et al. (2006) study in the cross maze paradigm could be interpreted as an impairment in extradimensional set shifting, it is also possible however that the effects observed are related to impairments in cognitive processes related to reversal learning (Hill et al., 2006), which 
would be consistent with our present findings. Concerning reversal learning abilities in other behavioral tasks, a clear involvement of the ECB system has been demonstrated. Acute THC treatment was found to impair performance in rats during the reversal phase of spatial learning in the Morris water maze (Boucher et al., 2009). Additionally, it has been shown that low doses of THC and URB597 impaired reversal learning, but not the acquisition or performance, of a two-odor discrimination task (Sokolic et al., 2011). In contrast with these findings, it has been reported that $\mathrm{CB} 1$ receptor knockout mice also displayed impaired reversal learning in a water maze task (Varvel and Lichtman, 2002). Therefore, the ECB system indeed appears to be important in adjusting behavioral strategies, however, the detailed direction of its modulations has not been completely clarified so far, although our present data demonstrate that increased availability of $\mathrm{CB} 1$ receptors in the $\mathrm{MPFC}$ impairs reversal learning abilities.

Notably, our present findings indicate an important role of $\mathrm{CB} 1$ receptors in the rat mPFC in reversal learning, but not in set shifting abilities. This finding is quite surprising since lesion studies reported that reversal learning strategies in the ASST depend mainly on the orbitofrontal cortex (McAlonan and Brown, 2003), whereas set shifting is mediated by the mPFC (Birrell and Brown, 2000). However, in contrast to these findings, other studies indicated that the rodent mPFC is indeed important for reversal learning abilities in different behavioral paradigms (Wolf et al., 1987; Joel et al., 1997; Li and Shao, 1998).

\section{REFERENCES}

American Psychiatric Association. (1994). Diagnostic and Statistical Manual of Mental Health Disorders (DSM $I V)$. Washington, DC: American Psychiatric Press.

Azad, S.C., Kurz, J., Marsicano, G., Lutz, B., Zieglgansberger, W., and Rammes, G. (2008). Activation of CB1 specifically located on GABAergic interneurons inhibits LTD in the lateral amygdala. Learn. Mem. 15, 143-152.

Barense, M. D., Fox, M. T., and Baxter, M. G. (2002). Aged rats are impaired on an attentional set-shifting task sensitive to medial frontal cortex damage in young rats. Learn. Mem. 9, 191-201.

Birrell, J. M., and Brown, V. J. (2000). Medial frontal cortex mediates perceptual attentional set shifting in the rat. J. Neurosci. 20, 4320-4324.

Bolla, K. I., Brown, K., Eldreth, D., Tate, K., and Cadet, J. L. (2002). Dose-related neurocognitive effects of marijuana use. Neurology 59, 1337-1343.

Boucher, A. A., Vivier, L., Metna-Laurent, M., Brayda-Bruno, L., Mons, N., Arnold, J. C., and Micheau, J. (2009). Chronic treatment with [delta]9tetrahydrocannabinol impairs spatial memory and reduces zif268 expression in the mouse forebrain. Behav. Pharmacol. 20, 45-55.

Colacicco, G., Welzl, H., Lipp, H. P., and Würbel, H. (2002). Attentional set-shifting in mice: modification of a dependent variation. Behav. Brain Res. 132, 95-102.

De Bruin, J. P. C. (1990). Social behaviour and the prefrontal cortex. Prog. Brain Res. 85, 485-497.

Dean, B., Sundram, S., Bradbury, R., Scarr, E., and Copolov, D. D. (2001). Studies on $[3 \mathrm{H}] \mathrm{CP}-55940$ binding in the human central nervous system: regional specific changes in density of cannabinoid-1 receptors associated with schizophrenia and cannabis use. Neuroscience 103, 9-15.

During, M. J., Young, D., Baer, K., Lawlor, P., and Klugmann, M. (2003). Development and optimization of adeno-associated virus vector transfer into the central nervous system. Methods Mol. Med. 76, 221-236.

Egerton, A., Allison, C., Brett, R. R., and Pratt, J. A. (2006). Cannabinoids and prefrontal cortical function: insights from preclinical studies. Neurosci. Biobehav. Rev. 30, 680-695.

Egerton, A., Brett, R. R., and Pratt, J. A. (2005). Acute delta9-tetrahydrocannabinol-induced deficits in reversal learning: neural correlates of affective inflexibility. Neuropsychopharmacology 30, 1895-1905.

File, S. E., and Hyde, J. R. G. (1978). Can social interaction be used to measure anxiety? Br. J. Pharmacol. 62, 19-24. rat paradigm, and evidence for strain-
In particular restricted lesions to either the prelimbic or the infralimbic area of the $\mathrm{mPFC}$, but not damage to the anterior cingulate area, was found to strongly inhibit reversal learning in a t-maze task (Li and Shao, 1998).

\section{CONCLUSION}

The present data indicate an important modulatory role for prefrontocortical CB1 receptors in emotional reactivity and arousal, social exploration and social withdrawal, as well as in cognitive flexibility. These findings might be relevant for neuropsychiatric disorders, since higher CB1 receptor expression levels in the PFC have been described post-mortem in schizophrenic patients (Dean et al., 2001; Zavitsanou et al., 2004). Schizophrenia is a chronic and severe brain disorder that has its symptomatic onset in early adulthood and affects multiple cognitive and behavioral functions, including behavioral flexibility and social skills (American Psychiatric Association, 1994). The AAV-technology applied in the present study therefore serves as an excellent tool to examine region-specific effects of alterations in CB1 receptor availability in the brain.

\section{ACKNOWLEDGMENT}

We would like to thank Claudia Klugmann and Christian Gluch for excellent technical assistance. Miriam Schneider was supported by DFG (SCHN 958/3-1). Matthias Klugmann was supported by a NSW OSMR Life Science Research Award.
Guggenhuber, S., Monory, K., Lutz, B., and Klugmann, M. (2010). AAV vector-mediated overexpression of CB1 cannabinoid receptor in pyramidal neurons of the hippocampus protects against seizure-induced excitoxicity. PLoS ONE 5, e15707. doi: 10.1371/journal.pone.0015707

Haller, J., Bakos, N., Szirmay, M., Ledent, C., and Freund, T. F. (2002). The effects of genetic and pharmacological blockade of the CB1 cannabinoid receptor on anxiety. Eur. J. Neurosci. 16, 1395-1398.

Haller, J., Varga, B., Ledent, C., Barna, I. and Freund, T. F. (2004). Contextdependent effects of CB1 cannabinoid gene disruption on anxiety-like and social behaviour in mice. Eur. J. Neurosci. 19, 1906-1912.

Herkenham, M., Lynn, A. B., Little, M. D., Johnson, M. R., Melvin, L. S., De Costa, B. R., and Rice, K. C. (1990). Cannabinoid receptor localization in brain. Proc. Natl. Acad. Sci. U.S.A 87, 1932-1936.

Hill, M. N., Froese, L. M., Morrish, A. C., Sun, J. C., and Floresco, S. B. (2006). Alterations in behavioral flexibility by cannabinoid CB1 receptor agonists and antagonists. Psychopharmacology (Berl.) 187, 245-259.

Holmes, A., and Wellman, C. L. (2009). Stress-induced prefrontal reorganization and executive dysfunction in rodents. Neurosci. Biobehav. Rev. 33, 773-783.

Jaferi, A., and Bhatnagar, S. (2007). Corticotropin-releasing hormone receptors in the medial prefrontal cortex regulate hypothalamic-pituitaryadrenal activity and anxiety-related behavior regardless of prior stress experience. Brain Res. 1186, 212-223. Joel, D., Weiner, I., and Feldon, J. (1997). Electrolytic lesions of the medial prefrontal cortex in rats disrupt performance on an analog of the Wisconsin card sorting test, but do not disrupt latent inhibition: implications for animal models of schizophrenia. Behav. Brain Res. 85, 187-201.

Klugmann, M., Leichtlein, C. B., Symes, C. W., Serikawa, T., Young, D., and During, M. J. (2005a). Restoration of aspartoacylase activity in CNS neurons does not ameliorate motor deficits and demyelination in a model of Canavan disease. Mol. Ther. 11, 745-753.

Klugmann, M., Symes, C. W., Leichtlein, C. B., Klaussner, B. K., Dunning, J., Fong, D., Young, D., and During, M. J. (2005b).AAV-mediated hippocampal expression of short and long Homer 1 proteins differentially affect cognition and seizure activity in adult rats. Mol. Cell. Neurosci. 28, 347-360.

Klugmann, M., and Szumlinski, K. K. (2008). Targeting Homer genes using adeno-associated viral vector: lessons 
learned from behavioural and neurochemical studies. Behav. Pharmacol. 19, 485-500.

Lafourcade, M., Elezgarai, I., Mato, S., Bakiri, Y., Grandes, P., and Manzoni, O. J. (2007). Molecular components and functions of the endocannabinoid system in mouse prefrontal cortex. PLoS ONE 2, e709. doi: 10.1371/journal. pone.0000709

Lane, S. D., Cherek, D. R., Tcheremissine, O. V., Steinberg, J. L., and Sharon, J. L. (2007). Response perseveration and adaptation in heavy marijuanasmoking adolescents. Addict. Behav. 32, 977-990.

Laviolette, S. R., and Grace, A. A. (2006). Cannabinoids potentiate emotional learning plasticity in neurons of the medial prefrontal cortex through basolateral amygdala inputs. $J$. Neurosci. 26, 6458-6468.

Li, L., and Shao, J. (1998). Restricted lesions to ventral prefrontal subareas block reversal learning but not visual discrimination learning in rats. Physiol. Behav. 65, 371-379.

Lominac, K. D., Oleson, E. B., Pava, M., Klugmann, M., Schwarz, M. K., Seeburg, P. H., During, M. J., Worley, P. F., Kalivas, P. W., and Szumlinski, K. K. (2005). Distinct roles for different Homer1 isoforms in behaviors and associated prefrontal cortex function. J. Neurosci. 25, 11586-11594.

Mailleux, P., and Vanderhaeghen, J. J. (1992). Distribution of neuronal cannabinoid receptor in the adult rat brain: a comparative receptor binding radioautography and in situ hybridization histochemistry. Neuroscience 48, 655-668.

Marsicano, G., and Lutz, B. (1999). Expression of the cannabinoid receptor CB1 in distinct neuronal subpopulations in the adult mouse forebrain. Eur. J. Neurosci. 11, 4213-4225.

Massa, F., Mancini, G., Schmidt, H., Steindel, F., Mackie, K., Angioni, C., Oliet, S. H., Geisslinger, G., and Lutz, B. (2010). Alterations in the hippocampal endocannabinoid system in dietinduced obese mice. J. Neurosci. 30, 6273-6281.

McAlonan, K., and Brown, V. J. (2003). Orbital prefrontal cortex mediates reversal learning and not attentional set shifting in the rat. Behav. Brain Res. 146, 97-103.

Moreira, F. A., Grieb, M., and Lutz, B. (2009). Central side-effects of therapies based on CB1 cannabinoid receptor agonists and antagonists: focus on anxiety and depression. Best Pract. Res. Clin. Endocrinol. Metab.23, 133-144.

Moreira, F.A., Kaiser, N., Monory, K., and Lutz, B. (2008). Reduced anxiety-like behaviour induced by genetic and pharmacological inhibition of the endocannabinoid-degrading enzyme fatty acid amide hydrolase (FAAH) is mediated by $\mathrm{CB} 1$ receptors. Neuropharmacology 54, 141-150.

O’Shea, M., Singh, M. E., McGregor, I. S., and Mallet, P. E. (2004). Chronic cannabinoid exposure produces lasting memory impairment and increased anxiety in adolescent but not adult rats. J. Psychopharmacol. 18, 502-508.

Owen, A. M., Roberts, A. C., Polkey, C. E., Sahakian, B. J., and Robbins, T. W. (1991). Extra-dimensional versus intra-dimensional set shifting performance following frontal lobe excisions, temporal lobe excisions or amygdalo-hippocampectomy in man. Neuropsychologia 29, 993-1006.

Pattij, T., Wiskerke, J., and Schoffelmeer, A. N. M. (2008). Cannabinoid modulation of executive functions. Eur. J. Pharmacol. 585, 458-463.

Paxinos, G., and Watson, C. (1998). The Rat Brain in Stereotaxic Coordinates. San Diego, CA: Academic Press.

Richichi, C., Lin, E. J., Stefanin, D., Colella, D., Ravizza, T., Grignaschi, G., Veglianese, P., Sperk, G., During, M. J., and Vezzani,A. (2004). Anticonvulsant and antiepileptogenic effects mediated by adeno-associated virus vector neuropeptide $Y$ expression in the rat hippocampus. J. Neurosci. 24, 3051-3059.

Rubino, T., Realini, N., Castiglioni, C., Guidali, C., Vigano, D., Marras, E., Petrosino, S., Perletti, G., Maccarrone, M., Di Marzo, V., and Parolaro, D. (2008). Role in anxiety behavior of the endocannabinoid system in the prefrontal cortex. Cereb. Cortex 18, 1292-1301.

Rubino, T., Sala, M., Vigano, D., Braida, D., Castiglioni, C., Limonta, V., Guidali, C., Realini, N., and Parolaro, D. (2007). Cellular mechanisms underlying the anxiolytic effect of low doses of peripheral [delta]9-tetrahydrocannabinol in rats. Neuropsychopharmacology 32, 2036-2045.

Schneider, M., and Koch, M. (2002). The cannabinoid agonist WIN 55,212-2 reduces sensorimotor gating and recognition memory in rats. Behav. Pharmacol. 13, 29-37.
Schneider, M., and Koch, M. (2005). Deficient social and play behavior in juvenile and adult rats after neonatal cortical lesion: effects of chronic pubertal cannabinoid treatment. Neuropsychopharmacology 30 , 944-957.

Schneider, M., Schömig, E., and Leweke, F. M. (2008). Acute and chronic cannabinoid treatment differentially affects recognition memory and social behavior in pubertal and adult rats. Addict. Biol. 13, 345-357.

Shah, A. A., and Treit, D. (2003) Excitotoxic lesions of the medial prefrontal cortex attenuate fear responses in the elevated-plus maze, social interaction and shock probe burying tests. Brain Res. 969, 183-194.

Sieber, B. (1982). Influence of hashish extract on the social behaviour of encountering male baboons (Papio $c$. anubis). Pharmacol. Biochem. Behav. 17, 209-216.

Sieber, B., Frischknecht, H. R., and Waser, P.G. (1980). Behavioral effects of hashish in mice. I. Social interactions and nest-building behavior of mice. Psychopharmacology 70, 149-154.

Singewald, N., Salchner, P., and Sharp, T. (2003). Induction of c-Fos expression in specific areas of the fear circuitry in rat forebrain by anxiogenic drugs. Biol. Psychiatry 53, 275-283.

Sokolic, L., Long, L. E., Hunt, G. E., Arnold, J. C., and McGregor, I. S. (2011). Disruptive effects of the prototypical cannabinoid delta9tetrahydrocannabinol and the fatty acid amide inhibitor URB-597 on go/ no-go auditory discrimination performance and olfactory reversal learning in rats. Behav. Pharmacol. 22, 191-202.

Steiner, M. A., Marsicano, G., Wotjak, C. T., and Lutz, B. (2008). Conditional cannabinoid receptor type 1 mutants reveal neuron subpopulationspecific effects on behavioral and neuroendocrine stress responses. Psychoneuroendocrinology 33, 1165-1170.

Trezza, V., and Vanderschuren, L. J. M. J. (2008). Cannabinoid and opioid modulation of social play behavior in adolescent rats: differential behavioral mechanisms. Eur. Neuropsychopharmacol. 18, 519-530.

Valverde, O. (2005). Participation of the cannabinoid system in the regulation of emotional-like behaviour. Curr. Pharm. Des. 11, 3421-3429.
Van Ree, J. M., Niesink, R. J. M., and Nir, I. (1984). [delta]1-Tetrahydrocannabinol but not cannabidiol reduces contact and aggressive behavior of rats tested in dyadic encounters. Psychopharmacology (Berl.) 84, 561-565.

Varvel, S. A., and Lichtman, A. H. (2002). Evaluation of CB1 receptor knockout mice in the Morris water maze. J. Pharmacol. Exp. Ther. 301, 915-924.

Viveros, M. P., Marco, E. M., and File, S. E. (2005). Endocannabinoid system and stress and anxiety responses. Pharmacol. Biochem. Behav. 81, 331-342.

Waltereit, R., Japs, B., Schneider, M., de Vries, P., and Bartsch, D. (2011) Epilepsy and Tsc2 haploinsufficiency lead to autistic-like social deficit behaviors in rats. Behav. Genet. 41, 364-372.

Wolf, C., Waksman, D., Finger, S., and Almli, C. R. (1987). Large and small medial frontal cortex lesions and spatial performance of the rat. Brain Res. Bull. 18, 1-5.

Wood, J. N. (2003). Social cognition and the prefrontal cortex. Behav. Cogn. Neurosci. Rev. 2, 97-114.

Zavitsanou, K., Garrick, T., and Huang, X. F. (2004). Selective antagonist $[3 \mathrm{H}]$ SR141716A binding to cannabinoid $\mathrm{CB} 1$ receptors is increased in the anterior cingulate cortex in schizophrenia. Prog. Neuropsychopharmacol. Biol. Psychiatry 28, 355-360.

Conflict of Interest Statement: The authors declare that the research was conducted in the absence of any commercial or financial relationships that could be construed as a potential conflict of interest.

Received: 05 June 2011; paper pending published:20 June 2011; accepted:29June 2011; published online: 13 July 2011.

Citation: Klugmann M, Goepfrich A, Friemel CM and Schneider M (2011) AAVmediated overexpression of the $C B 1$ receptor in the MPFC of adult rats alters cognitive flexibility, social behavior, and emotional reactivity. Front. Behav. Neurosci. 5:37. doi: 10.3389/fnbeh.2011.00037

Copyright () 2011 Klugmann, Goepfrich, Friemel and Schneider. This is an open-access article subject to a non-exclusive license between the authors and Frontiers Media $S A$, which permits use, distribution and reproduction in other forums, provided the original authors and source are credited and other Frontiers conditions are complied with. 